\title{
Bestimmung der Aktivität der Lipoprotein-Lipase im Plasma nach Heparinverabreichung mit Hilfe der Bromsulphthaleinmethode
}

\author{
Von L. Kučerová, V. Hoentg, E. Fabian, M. Jirsa und A. Štork \\ Anis dem Forscbungslaboratorium fiur die Patbophysiologie der Blutbildung und der Leber an der I. Medizinischen Klinik (Vor- \\ stand: Prof. Dr. med. V. Hoenig, DrSc.) der mediqinischen Fakultät der Karls-Universität in Prag
}

(Eingegangen am 20. Januar 1966)

\begin{abstract}
Bei 23 gesunden Personen wurden im Nüchternplasma und im Plasma, das 10 Min. nach intravenöser Verabreichung von $50 \mathrm{mg}$ Heparin entnommen wurde, die Konzentration der unveresterten Fettsäuren und die Albuminämie bestimmt, letztere sowohl elektrophoretisch, als auch mit der Bromsulphthaleinmethode. Wir konnten bestätigen, daß nach Heparinverabreichung die Konzentration der unveresterten Fettsäuren ansteigt. Diese Fettsäuren binden sich an Albumin und bewirken so eine herabgesetzte Bindungsfähigkeit des Plasmaeiweißes für Bromsulphthalein. Die mit der Bromsulphthaleinmethode ermittelten Albuminämiewerte sind daher scheinbar herabgesetzt. Daraus geht hervor, daß die Bromsulphthaleinmethode bei erhöhtem Plasmaspiegel der unveresterten Fettsäuren zur Bestimmung der Albuminämie nicht verwendet werden kann.

Der Anstieg der Konzentration der unveresterten Fettsäuren nach Heparinverabreichung ist dem Anstieg der Extinktion einer Standardlösung von Bromsulphthalein und Plasma nach Heparinverabreichung dirckt proportional. Aus der Extinktionsänderung der Bromsulphthaleinlösung kann der Anstieg der unveresterten Fettsäuren im Plasma mit einem Fehler von $\pm 16,2 \%$ berechnet werden. Die Bromsulphthaleinmethode gestattet also, die Menge der bei der Klärungsreaktion freigewordenen unveresterten Fettsäuren zu bestimmen und indirekt so dic Aktivität der Lipoprotein-Lipase in vivo festzustellen.
\end{abstract}

23 Healthy persons were used to determine the concentrations of unesterified fatty acids and albuminaemia in resting plasma and in plasma taken $10 \mathrm{~min}$. after the intravenous injection of $50 \mathrm{mg}$. of heparin. Albuminaemia was measured both electrophoretically and by the bromosulphalein method. We showed that the concentration of unesterified fatty acids increased after the administration of heparin. These fatty acids became bound to albumin and thereby rendered the plasma protein less reactive with bromosulphalein. Thus the albumin value by the bromosulphalein method was apparently low. The bromosulphalein method for albuminaemia cannot therefore be used when the level of unesterified plasma fatty acids is increased.

After the administration of heparin, the increase in the concentration of free fatty acids is directly proportional to the increase in the extinction of a standard solution of bromosulphalein and plasma. The increase in the level of unesterified plasma fatty acids can be determined from the change in extinction of the bromosulphalein solution with an error of $\pm 16.2 \%$. Therefore, the bromosulphalein method permits the measurement of the unesterified fatty acids in the clearing reaction and, indirectly, the activity of the lipoprotein lipase in vivo.

Unveresterte Fettsäuren (UFS) sind im Blutplasma an Eiweiß gebunden, vorwiegend an Albumin (1-4). An Plasmaalbumin binden sich außerdem noch andere Stoffe $(5,6)$, und $z$ war sowohl endogene (Bilirubin, Gallensäuren) als auch exogene (Farbstoffe, Medikamente). Die Bindungsfähigkeit von Albumin gegenüber Farbstoffen kann zur Bestimmung der Albuminämie dienen (7-10). Die von uns verwendete Methode (9) beruht darauf, daß zu einer Standardlösung von Bromsulphthalein („BSP“) verdünntes Plasma hinzugefügt wird, das entsprechend der Albuminkonzentration eine bestimmte Menge Bromsulphthalein bindet und die Extinktion der Lösung verändert; die Farbintensität der Standardlösung sinkt umso mehr ab, je höher die Albuminämie ist. Mit Hilfe dieser Methode wird also lediglich jener Anteil von Albumin bestimmt, der fähig ist, Bromsulphthalein $\mathrm{zu}$ binden.

In einer früheren Arbeit (11) haben wir nachgewiesen, daß Fettsäuren eine größere Bindungsfähigkeit gegenüber Albumin aufweisen als Bromsulphthalein. Bromsulphthalein wird daher durch Fettsäuren aus seiner Bindung an Albumin verdrängt. Man könnte also voraussetzen, daß eine Vermehrung der unveresterten Fettsäuren im Plasma, wie dies bei der Klärungsreaktion nach intravenöser Verabreichung von Heparin der Fall ist $(12,13)$, bei der Bestimmung der Albuminämie mit Hilfe von Bromsulphthalein eine scheinbare Albumin- verminderung zur Folge hätte. Bei steigender Konzentration der unveresterten Fettsäuren, die sich an Albumin binden, würde nämlich der Albuminanteil, der fähig ist Bromsulphthalein zu binden, absinken, und somit würde sich bei der Bromsulphthaleinmethode eine geringere Albuminämie ergeben.

In dieser Arbeit stellten wir uns daher die Frage: 1. welchen Einfluß übt die Vermehrung der unveresterten Fettsäuren bei der Klärungsreaktion auf die mit der Bromsulphthaleinmethode ermittelten Albuminämiewerte aus; 2. ist es möglich, diese einfache Methode zur Bestimmung des Anstiegs der unveresterten Fettsäuren bei der Klärungsreaktion in vivo anzuwenden ?

\section{Methodik}

Gesunden nüchternen Personen wurde Blut in Heparin entnommen und mit der gleichen Kanüle $50 \mathrm{mg}$ Heparin (Fa. SPOFA) intravenös injiziert; nach $10 \mathrm{Min}$. wurde erneut Blut in Heparin entnommen. Das Blut wurde bei $0^{\circ}$ aufbewahrt und so schnell wie möglich vẹrarbeitet.

In beiden Plasmaproben wurde der Spiegel der unveresterten Fettsäuren kolorimetrisch nach Nováx (14) bestimmt und die Albuminkonżentration einerseits elektrophoretisch, andererseits mittels der Bromsulphthaleinmethode (9) untersucht. Bei dieser Methode fügt man $0,5 \mathrm{~m} l$ Plasma, das mit Wasser im Verhältnis $1: 20$ verdünnt worden war, zu 4,5 ml 1,4 mg-proz. Standardlösung von Bromsulphthalein in Boratpuffer mit pH 9,1. Die Extinktion der Bromsulphthaleinlösung wird am Pulfrichphotometer bei $580 \mathrm{~m} \mu$ gegen Wasser gemessen. Die Kalibrierung wurde mit menschlichem 


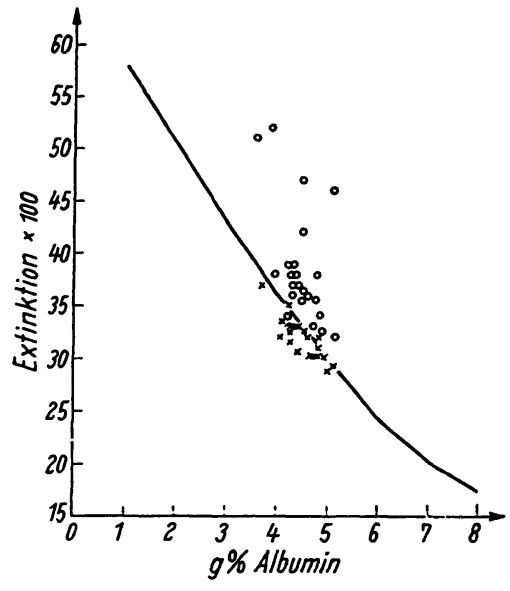

Abb. 1

Die Beziehung der elektrophoretisch bestimmten Albuminämie zu der mittels Bromsulphthaleinmethode festgestellten Albuminämie im Plasma vor und nach Heparininjektion

Das Diagramm veranschaulicht eine Kurve von 2 bis 8-proz. Albumin, mittels Bromsulphthaleinmethode bestimmt. Die mit $\times$ bezeichneten bezeichneten Punkte sind Werte, die im 10 Minuten nach Heparininjektion entnommenen Plasma gemessen wurden

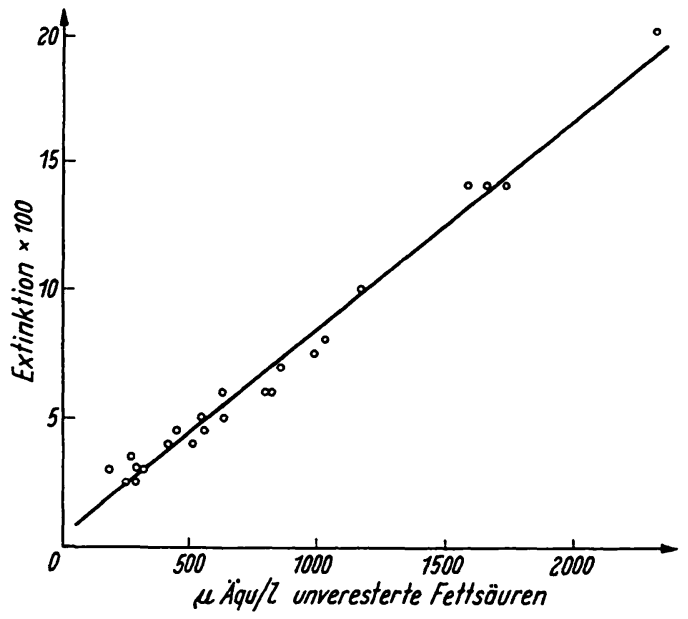

Abb. 2

Die Beziehung zwischen dem Konzentrationsanstieg der unveresterten Fettsäuren und der Extinktionsänderung des Bromsulphthalein-Plasma-Gemisches nach Heparininjektion

Die Regressionskurve weist auf eine äußerst enge lineare Abhängigkeit beider Werte hin ( $r s=4,65>3$ ) lyophilisiertem Albumin (1 Jahr alt) durchgeführt, wobei die wäßr. 2-6-proz. Albuminlösungen mit Wasser im Verbältnis 1:20 verdünnt wurden und davon je $0,5 \mathrm{~m} l \mathrm{zu} 4,5 \mathrm{~m} l$ Standardlösung von Bromsulphthalein hinzugefügt wurden.

\section{Ergebnisse}

Auf diese Art wurden 23 gesunde Personen (13 Frauen und 10 Männer) im Alter von 20 bis 73 Jahren untersucht; der Altersdurchschnitt betrug 37,7 Jahre.

Die durchschnittliche Konzentration der unveresterten Fettsäuren im Plasma des nüchternen Probanden (Tab. 1) betrug $473 \pm 168,2 \mu \mathrm{A} q / /$; nach der Heparininjektion stieg sie im Durchschnitt auf $1278 \pm 601,7$ $\mu \ddot{A} q / l$ an. Der Plasmaspiegel der unveresterten Fettsäuren erhöhte sich also im Durchschnitt um $803 \pm 181,4$ $\mu$ Äq/l. Die Extinktion $(\times 100)$ der Bromsulphthaleinlösung mit Nüchtern-Plasma betrug durchschnittlich $31,9 \pm 1,7$; mit Plasma nach Heparinverabreichung stieg sie im Durchschnitt auf $38,6 \pm 5,32$. Die Extinktion ( $\times 100)$ der Bromsulphthaleinlösung stieg also im Durchschnitt um 6,7 \pm 4,6 an. Die elektrophoretisch ermittelte Albuminkonzentration unterlag nur geringen und nichtkonstanten Veränderungen.

Die Werte für Plasmaalbumin beim nüchternen Probanden - bestimmt mittels Bromsulphthalein - sind mit den Ergebnissen unserer früheren Arbeit (9) identisch und stehen mit der elektrophoretisch ermittelten Albuminkonzentration im Einklang. Demgegenüber sind die mittels Bromsulphthalein im Plasma nach Heparinverabreichung gefundenen Werte weitaus niedriger (Abb. 1). Nach Heparininjektion steigt also zugleich mit dem Spiegel der unveresterten Fettsäuren die Extinktion der Lösung Bromsulphthalein + Plasma bei praktisch unveränderter Albuminkonzentration an - als Folge der partiellen Blockierung von Albumin durch die Fettsäuren, die Bromsulphthalein aus seiner Bindung an Albumin verdrängt haben.

Aus Abbildung 2 ist ersichtlich, daß zwischen der Veränderung der Konzentration der unveresterten Fettsäuren und der Veränderung der Farbintensität der

Tab. 1

Der Plasmaspiegel der unveresterten Fettsäuren und die einerseits mit der BSP-Methode, andererseits elektrophoretisch bestimmte Albuminämie vor und nach Heparininjektion bei gesunden Personen

\begin{tabular}{|c|c|c|c|c|c|c|c|c|c|c|c|c|}
\hline Nr. & Alter & Geschlecht & \multicolumn{2}{|c|}{$\begin{array}{l}\text { UFS in } \mu \AA \AA q / l \\
\text { Heparin } \\
\text { vor }{ }_{\text {nach }}\end{array}$} & \multicolumn{2}{|c|}{$\begin{array}{l}\text { Albuminämie-BSP } \\
\text { Heparin } \\
\text { vor nach } \\
\text { Extinktion } \cdot 100\end{array}$} & $\begin{array}{l}\text { elektro } \\
\text { vor }\end{array}$ & $\begin{array}{l}\text { n } \\
\text { nach }\end{array}$ & \multicolumn{2}{|c|}{$\begin{array}{cc}\text { Anstieg von } \\
\text { BSP } & \text { UFS } \\
\mathrm{E} \cdot 100 & \mu \AA \mathrm{A} / l\end{array}$} & \multicolumn{2}{|c|}{$\frac{\text { Anstieg der UFS }}{\text { Anstieg des BSP }}$} \\
\hline $\begin{array}{r}1 \\
2 \\
3 \\
4 \\
5 \\
6 \\
7 \\
8 \\
9 \\
10 \\
11 \\
12 \\
13 \\
14 \\
15 \\
16 \\
17 \\
18 \\
19 \\
20 \\
21 \\
22 \\
23\end{array}$ & $\begin{array}{l}20 \\
20 \\
25 \\
54 \\
52 \\
48 \\
27 \\
35 \\
24 \\
50 \\
29 \\
35 \\
53 \\
53 \\
33 \\
38 \\
26 \\
31 \\
73 \\
42 \\
16 \\
41 \\
43\end{array}$ & $\begin{array}{l}\mathbf{w} \\
\mathbf{w} \\
\mathbf{w} \\
\mathbf{w} \\
\mathbf{w} \\
\mathbf{w} \\
\mathbf{w} \\
\mathbf{w} \\
\mathbf{w} \\
\mathbf{w} \\
\mathbf{w} \\
\mathbf{w} \\
\mathbf{w} \\
\mathbf{m} \\
\mathbf{m} \\
\mathbf{m} \\
\mathbf{m} \\
\mathbf{m} \\
\mathrm{m} \\
\mathbf{m} \\
\mathbf{m} \\
\mathbf{m} \\
\mathbf{m}\end{array}$ & $\begin{array}{l}200 \\
150 \\
440 \\
565 \\
520 \\
465 \\
400 \\
500 \\
555 \\
980 \\
710 \\
475 \\
440 \\
385 \\
455 \\
360 \\
530 \\
330 \\
395 \\
600 \\
320 \\
600 \\
520\end{array}$ & $\begin{array}{r}835 \\
440 \\
1300 \\
980 \\
970 \\
1105 \\
670 \\
2165 \\
1545 \\
1275 \\
1880 \\
725 \\
1000 \\
2130 \\
1485 \\
1180 \\
715 \\
845 \\
2725 \\
2295 \\
880 \\
925 \\
1320\end{array}$ & $\begin{array}{l}33 \\
33 \\
28,5 \\
33 \\
32 \\
30 \\
32,5 \\
37 \\
30,5 \\
35 \\
32,5 \\
31,5 \\
33,5 \\
33 \\
30 \\
33 \\
30 \\
30 \\
32 \\
32 \\
31 \\
29 \\
32\end{array}$ & $\begin{array}{l}38 \\
35,5 \\
35,5 \\
37 \\
36,5 \\
35 \\
36 \\
51 \\
38 \\
37 \\
42,5 \\
34 \\
38 \\
47 \\
38 \\
39 \\
33 \\
34 \\
52 \\
46 \\
36 \\
32 \\
38\end{array}$ & $\begin{array}{l}4,30 \\
4,23 \\
5,01 \\
4,42 \\
4,63 \\
4,95 \\
4,24 \\
3,68 \\
4,39 \\
4,28 \\
4,56 \\
4,26 \\
4,09 \\
4,30 \\
4,72 \\
4,40 \\
4,74 \\
4,79 \\
4,06 \\
4,81 \\
4,78 \\
5,18 \\
4,55\end{array}$ & $\begin{array}{l}4,36 \\
4,47 \\
4,78 \\
4,42 \\
4,48 \\
4,85 \\
4,30 \\
3,58 \\
4,30 \\
4,44 \\
4,50 \\
4,22 \\
3,89 \\
4,50 \\
4,75 \\
4,28 \\
4,69 \\
4,79 \\
3,91 \\
5,10 \\
4,54 \\
5,10 \\
4,33\end{array}$ & $\begin{array}{c}5 \\
2,5 \\
7 \\
4 \\
4,5 \\
5 \\
3,5 \\
14 \\
7,5 \\
2 \\
10 \\
2,5 \\
4,5 \\
14 \\
8 \\
6 \\
3 \\
4 \\
20 \\
14 \\
5 \\
3 \\
6\end{array}$ & $\begin{array}{r}635 \\
290 \\
860 \\
415 \\
450 \\
630 \\
270 \\
1665 \\
990 \\
295 \\
1170 \\
250 \\
560 \\
1745 \\
1030 \\
820 \\
185 \\
515 \\
2330 \\
1695 \\
550 \\
325 \\
800\end{array}$ & $\begin{array}{r}127 \\
116 \\
123 \\
104 \\
100 \\
126 \\
77 \\
119 \\
132 \\
148 \\
117 \\
100 \\
124 \\
125 \\
129 \\
137 \\
62 \\
129 \\
116 \\
121 \\
110 \\
108 \\
133\end{array}$ & . \\
\hline
\end{tabular}


Bromsulphthaleinlösung eine äußerst enge lineare $A b$ bängigkeit bestcht (Korrelationskoeffizient $r=0,99$; rs $=4,65>3)$. Um dic Veränderung der Konzentration der unveresterten Fettsäuren aus der Extinktionsänderung der Bromsulphthaleinlösung berechnen zu können, stellten wir fest, um wieviel die Konzentration der unveresterten Fettsäuren anstieg, wenn sich die Extinktion $(x 100)$ der Bromsulphthaleinlösung um 1 erhöhte, und zwar nach der Formel:

Anstieg der unveresterten Fettsäuren in $\mu \AA \mathrm{A} q / l$

Anstieg der Extinktion ( $\times 100)$ der Bromsulphthalcinlösung

Aus Tabelle 1 ist ersichtlich, daß ein Extinktionsanstieg der Bromsulphthaleinlösung um 1 einer Konzentrationserhöhung der unveresterten Fettsäuren um durchschnittlich $117 \pm 19 \mu$ Äq/l entspricht. Bei einem Extinktionsanstieg der Bromsulphthaleinlösung um mehr als 5, wobei sich die Konzentration der unveresterten Fettsäuren um mehr als $600 \mu \mathrm{A} q / l$ erhöhte, ist dic Streuung der Werte weitaus geringer; ein Extinktionsanstieg der Bromsulphthaleinlösung um 1 entspricht in diesem Falle einem Konzentrationsanstieg der unveresterten Fettsäuren um durchschnittlich $125 \pm 6$ $\mu \ddot{\mathrm{A}} \mathrm{q} / l$. Die Veränderung der Extinktion der Bromsulphthaleinlösung ist dann praktisch das Maß des Anstiegs der unveresterten Fettsäuren. Die Berechnung der Konzentrationsänderung der unveresterten Fettsäuren ist also mit einem Fehler von $\pm 16,2 \%$ belastet, während sie bei einem Anstieg über $600 \mu \mathrm{Äq} / l$ nur einen Fehler von $\pm 4,8 \%$ aufweist.

\section{Diskussion}

Unsere Befunde weisen darauf hin, daß bei der Erhöhung des Plasmaspiegels der unveresterten Fettsäuren während der Klärungsreaktion nach Heparinverabreichung eine Erhöhung der Extinktion der Bromsulphthalein-Standardlösung, der dieses Plasma zugefügt worden war, erfolgt. Obwohl sich dabei die tatsächliche Albuminkonzentration nicht verändert, erhalten wir hier mit Hilfe der Bromsulphthaleinmethode herabgesetzte Albuminämiewerte. Dieser Befund spricht - im Einklang mit den Ergebnissen unserer früheren Untersuchung in vitro - für die Verdrängung des Bromsulphthaleins aus seiner Bindung an Albumin durch die Fettsäuren. Bei erhöhter Plasmakonzentration der unveresterten Fettsäuren ist daher die Bromsulphthaleinmethode für die Bestimmung der Albuminämie nicht verwendbar. Aus den Ergebnissen geht weiter hervor, daß mit der Bromsulphthaleinmethode der Anstieg des Plasmaspiegels der unveresterten Fettsäuren mit einem Fehler von $\pm 16,2 \%$, bei höherem Anstieg mit einem Fehler von $\pm 4,8 \%$ festgestellt werden kann.
Diese einfache Methode, die hinreichend genau die bei der Klärungsreaktion freigewordenen unveresterten Fettsäuren bestimmt, kann also die anspruchsvollere Bestimmung der Konzentration der unveresterten Fettsäuren im Plasma ersetzen. Da der Anstieg der unveresterten Fettsäuren nach Heparinverabreichung vornehmlich von der Aktivierung der Lipoprotein-Lipase abhängt $(12,15)$, ist cs möglich, sich mittcls der Bromsulphthaleinmethode über die Aktivität dieses Enzyms in vivo $\mathrm{zu}$ informieren.

Im Einklang mit unseren Befunden beobachtete THERKELSEN (16), daß die intravenöse Verabreichung von Heparin bei Hunden Veränderungen in der Spektralabsorption des Bromsulphthalein-Serum-Gemisches hervorruft, die er der Bindung von freigewordenen unveresterten Fettsäuren an Albumin zuschreibt, wenn er auch deren Konzentration im Plasma nicht zugleich bestimmte.

ZEMPLENYI (17) untersuchte die Bindungsfähigkeit der Eiweißkörper des Blutserums anhand der Adsorption von Kongorot an Filtrierpapicr. Das Prinzip der von ihm verwendeten Methode beruht darauf, daß aus Filtrierpapier mit physiologischer Lösung nur der Anteil von Kongorot eluiert werden kann, der an Albumin gebunden ist. Die Fettsäuren, die Kongorot aus dieser Bindung verdrängen, vermindern so das eluierbare Quantum von Kongorot. Nach intravenöser Heparinverabreichung ändert sich die Bindungsfähigkeit des Bluteiweißes für Kongorot als Folge der Bindung der freigewordenen unveresterten Fettsäuren an Albumin. Die Methode erwies sich als ein empfindlicher Indikator der Klärungsreaktion, aber der Anstieg der unveresterten Fettsäuren wurde quantitativ nicht bewertet.

Mit einer ähnlichen Problematik befaßte sich MAY (10), der feststellte, daß die Zugabe von Oleinsäure das Spektrum eines Eosin-Albumin-Gemisches veränderte, und der diese Eosinmethode ebenso wie andere Autoren $(18,19,20)$ zur Bestimmung der Aktivität der Lipoprotein-Lipase in vitro verwendete. Postheparin-Rattenplasma wurde mit Albumin, Eosin und lipämischem Plasma oder einer Fettemulsion inkubiert. Das Spektrum des Eosins veränderte sich während der Inkubation als Folge des Anstiegs der Fettsäuren und ihrer Bindung an Albumin proportional der Aktivität der LipoproteinLipase. Ein Nachteil dieser Methode gegenüber der Bromsulphthaleinmethode besteht darin, daß bei Verwendung von Eosin nicht nur eine Intensitätsänderung, sondern auch eine Farbänderung der Lösung erfolgt, so $\mathrm{da} B$ an zwei Filtern abgelesen werden muß und die Bewertung schwieriger wird.

\section{Literatur}

1. Axenfeld, H., Wien. klin. Wschr. 68, 296 (1956). - 2. Davis, B. D. und R. J. Dubos, J. Exper. Med. 86, 215 (1947). - 3. GoodMAN, D. S. und E. SFiAfrur, J. Amer. chem. Soc. 81, 364 (1959). 4. Rerchl, D., Čs. Fysiol. 9, 338 (1960). - 5. Bennhold, H., Protides of the Biological Fluids. Elsevier Publ. Comp., Amsterdam (1961). - 6. Bennmold, H. und H. OTt, Der Stofftransport, in:
Hdb. Allg. Path. Band V/1. Springer-Verlag, Berlin-GöttingenHeidelberg (1961). - 7. BLondheim, S. H., J. Laborat. Clin. Med., S. Louis 45, 740 (1955). - 8. BrACKEN, J. S. und I. M. KLoTz, Amer. J. Clin. Path. 23, 1055 (1953). - 9. JirsA, M. und V. Hoentg, Cas. Lčk. čcsk. 103, 267 (1964). - 10. MAY, P., Rev. franc. d'Études clin. et biol. 3, 53 (1958). - 11. Kučerovi, L., V. 
Hoenig, M. Jirsa und E. Fabian, Čas. Lěk. česk., im Druck. 12. Fabian, E., L. Kučerová, A. Śtork und J. Śponarovi, Plzcňský sbornik, im Druck. - 13. Sprrzer, J. J., IIIrd Internat. Conf. on Biochemical Problems of Lipids (Brüssel, 1956); Klaase der Wetenschapen, 1957 Belgie. - 14. Novík, M., J. Lipid. Res. 6, 456 (1965). - 15. Engelberg, H., Amer. J. Cardiol. 14, 8 (1964). - 16. Therkelsen, A. J., Scand. J. Clin. Laborat.
Invest. 9, 156 (1957). - 17. Zemplényr, T., Cas. lěk. česk. 97, 1230 (1958). - 18. LeBreton, E., Ch. Frayssinet und P. May, Bull. Acad. suisse sc. méd. 13, 229 (1957). - 19. Frayssinet, C. und P. MAY IIIrd Internat. Conf. on Biochemical Problems of Lipids (Brüssel, 1956), Klaase der Wetenschapen. 1957 Belgie. - 20. Max, P., C. R. Acad. Sci. Paris 245, 2551 (1957).

Dr. L. Kučerová

I. Medizinische Klinik der Karls-Universität Prag 2, U nemocnice 2

\title{
Determination of citric acid in urine and in serum
}

\author{
By I. Antener, L. Vuataz, A. Bruschi and M. Kaeser \\ From the Nestle Research Laboratories (Director Dr. R. H. Egli) Vevey, Switzerland
}

(Eingegangen am 26. Januar 19.66)

\begin{abstract}
A method is described for the determination of citric acid in the routine clinical investigation of urine and serum. It is based on a combination of the methods of GEY, LANE and CHEN. The method has been used for several years and has been completed by a statistical analysis.
\end{abstract}

Es wird die Beschreibung einer Zitronensäurebestimmungsmethode im Harn und Serum, die sich gut zu Reihenuntersuchungen in der Klinik eignet, gegeben. Sie beruht auf der Kombination der Methoden von GEY undLANE und CHEN. Während mehrerer Jahre wurde diese Methode angewendet und durch eine Statistik ergänzt.

Recent progress in clinical chemical methods has shown the importance of citric acid in the different metabolic pathways. Its storage and release is influenced by vitamin $\mathrm{D}$ and parathormone, and it is a metabolite of the Krebs cycle. Citric acid is also a bone component. Moreover, the secretion and tubular reabsorption of renal citric acid are two problems which remain unsolved. Determinations of serum and urinary citric acid are thus not only of interest in the different bone diseases, but also in certain kidney and liver diseases.

The clinical laboratory therefore needs a method for the determination of citric acid in urine and in serum, which is precise, rapid and not too complicated. This paper describes such a method which we have used in our laboratory. Our six years of experience have shown it to be capable of fulfilling all the necessary requirements of the clinical laboratory. Above all, we would like to emphasize its accuracy which permits, for example, the differentiation of certain diseases by analysis of the citric acid levels in serum.

We have found many methods for the determination of citric acid in the literature, most of them based on the conversion of citric acid into pentabromacetone $(\mathrm{Pba})$, with various modifications for its oxidation and extraction (2-12). We have retained the methods of GEY, and LANE and CHEN. After trying both, we decided that a judicious combination of the two would permit us to shorten the time required for the determination without sacrificing its sensitivity. We therefore kept LANE et al's sequence of oxidation at low temperature and the subsequent reduction of the excess permanganate by hydrogen peroxide. We then substituted GEY's technique for extraction with petroleum ether, elimination of traces of acid, and formation of the $\mathrm{Pba}$-thiourea-borax complex.
Thus the combination of the two methods permits us to shorten the time required for the determination without affecting the accuracy of the results. By adopting GEx's method of extraction, we have eliminated certain inherent errors of the NaI-ethanol extraction, for example the influence of light, temperature and the possible contamination of the NaI-Pba-ethanol mixture by acids, which would have to be eliminated by washing with water and the phosphate buffer. Furthermore, the $\mathrm{Pba}$-thioureaborax complex is not affected by the presence of $\beta$-hydroxybutyric acid, acetone and succinic acid, even in pathological quantities. The $\mathrm{Pba}-\mathrm{NaI}$-ethanol reaction is sensitive to the aforementioned compounds, as well as to aspartic acid.

\section{Method}

\section{Principle}

Citric acid is converted into pentabromacetone ( $\mathrm{Pba})$ in acidic solution and at low temperature by a reagent consisting of potassium bromide and potassium permanganate. The excess potassium permanganate is reduced by hydrogen peroxide. The $\mathrm{Pba}$ formed is extracted with light petroleum, the extract is washed, and the $\mathrm{Pba}$ re-extracted with a solution of sodium borate thiourea. The resulting complex has an absorption maximum at $450 \mathrm{~m} \mu$.

\section{Reagents}

10\% Trichloroacetic Acid: $10 \mathrm{~g}$ trichloroacetic acid (p. a.) Grade $\mathrm{A}+90 \mathrm{~m} l$ distilled water.

Sulfuric Acid $9 \mathrm{~N}: 252 \mathrm{ml}$ conc. $\mathrm{H}_{2} \mathrm{SO}_{4}$ (density 1,84, Grade A) to $1 l$ with distilled water.

Standard Citric Acid Solution: Dissolve $100 \mathrm{mg}$ anhydrous citric acid or $109,8 \mathrm{mg}$ citric acid $1 \mathrm{H}_{2} \mathrm{O}$ in $10 \mathrm{ml} 9 \mathrm{~N} \mathrm{H}_{2} \mathrm{SO}_{4}$ and dilute to. 